\title{
Disturbance Observer Based Control of Multirotor Helicopters Based on a Universal Model with Unstructured Uncertainties
}

\author{
Ye Xie, Yunfeng Cao, Biao Wang, and Meng Ding \\ Nanjing University of Aeronautics and Astronautics, Nanjing 210016, China \\ Correspondence should be addressed to Yunfeng Cao; cyfac@nuaa.edu.cn
}

Received 15 October 2014; Revised 3 December 2014; Accepted 10 February 2015

Academic Editor: Meng J. Er

Copyright (C) 2015 Ye Xie et al. This is an open access article distributed under the Creative Commons Attribution License, which permits unrestricted use, distribution, and reproduction in any medium, provided the original work is properly cited.

To handle different perspectives of unstructured uncertainties, two robust control techniques on the basis of a universal model are studied in this paper. Rather than building a model only applicable to a specific small-scale multirotor helicopter (MHeli), the paper proposes a modeling technique to develop a universal model-framework. Particularly, it is straightforward to apply the universal model to a certain MHeli because the contribution and allocation matrix is proposed in the model-framework. Based on the model uncertainties, the load perturbation of the rotor is the primary focus due to its indispensable importance in the tracking performance. In contrast to the common methods, it is proposed to take this unstructured uncertainty in that external disturbance and designs disturbance observer (DOB). In addition, a class of lead-compensator is specifically designed as for compensating phase lag induced by DOB. Compared with $H_{\infty}$ loop-shaping, greater robust tracking performance on rejecting load perturbation could be achieved as a tradeoff between robust stability and tracking performance which is successfully avoided with DOB-based control strategy.

\section{Introduction}

The small-scale multirotor helicopter (MHeli) is emerging as a sort of popular unmanned aerial vehicles (UAVs) for its great value on autonomy and intelligence research. In comparison with traditional small-scale aircraft, beside the construction simplicity and maintenance, the maneuverability, the vertical taking off and landing (VTOL) capability, and the hovering ability of small-scale helicopters indicate the extreme potential for coordinated reconnaissance, tracking, searching, and so forth [1]. In contrast to the above superiority, trajectory-following precision of the small-scale MHeli is severely affected by various model uncertainties, especially the load perturbation of multiple rotors which is a certain classic unstructured uncertainty [2].

The MHeli is generally defined as a class of UAVs whose movements are derived from changes in the angular speed of the rotors, so the dynamics of a MHeli is largely dependent on the dynamics of its rotors. If a MHeli reaches a stable hovering situation, the angular speed of rotor at steady state would mainly lie on the input of rotor. However, when a MHeli performs high maneuvers, the dynamics of the rotor would be widely divergent with the hovering one because of totally different loading effects. The abovementioned phenomenon is known as load perturbation or rotor damping, and since it would directly affect the rotor efficiency, then the tracking performance of the MHeli would be largely impacted.

To recover full maneuverability of a MHeli with model uncertainties, numerous nonlinear controls are employed to achieve aggressive maneuvers with high performance. Though many of these successfully handle the problem that some kinds of nonlinear control methods are highly dependent on the exact dynamic model $[3,4]$, the tracking performance generally trades off asymptotic stability with stability margin. On the other hand, neural networks become popular as a method for handling unknown nonlinearities. In [5], a feed-forward neural network is used to approximate the unknown nonlinear dynamics so it does not require any prior information on model uncertainties. In contrast to its great theoretical value, the neural network control encounters huge difficulties with realization in embedded unmanned autonomous system.

In view of robust performance and implementation in practice, a category of robust controls is preferred in 
research fields on small-scale MHeli. Work [6] guarantees stability and tracking performance at the same time by using a robust control term, yet not involved in any specific model uncertainty. In [7-9], the proposed adaptive or sliding mode approaches achieve asymptotic tracking of a quadrotor helicopter in the presence of parametric uncertainties and measurement noise. Reference [10] describes the application of a model reference adaptive control based on Lyapunov stability arguments and offers increased robustness to mitigate the effects of a loss-of-thrust anomaly. However, in both of these approaches only parametric uncertainties are considered, whereas unstructured uncertainties are not handled in their controller designs. Moreover, in the aforementioned methods, the tradeoff between robust stability and tracking performance is not fully discussed in their robust analysis of the closed-loop system.

The $H_{\infty}$ optimization has been used to successfully trade robustness for unstructured uncertainties. For minimizing the potential difficulties of MIMO loop-shaping, formula [11] employs a linear quadratic design to form the weight control and reaches good robustness to a class of generic uncertainty; however, it does not include uncertainty rejection concerning load perturbation. With simpler structure and less calculation, disturbance observer (DOB) satisfies the real-time requirement and has been very successful and has been widely employed in control system with high precision and accuracy requirements. Works [12-14] employ the complicated nonlinear DOB to design robust controller for quadrotors and recover the system performance under parameter uncertainties and disturbance. However, the above methods are not involved in unstructured uncertainties and the final controller seems too complex to be put into practice. References $[15,16]$ perform the task of successfully rejecting variable payloads and their controllers are verified by simulation and experiment. It is noted that only a few papers introduce payload problems into DOB design, in absence of the further study of relationship between robust stability and tracking performance.

The paper devotes itself to handling the problems of MHeli's susceptible trajectory tracking performance mainly caused by load perturbation and proposes the better robust control which can effectively evade tradeoff between robust stability and tracking performance, based on a brand-new universal model. This paper is organized as follows: Section 2 presents the universal model of MHeli by proposing a modeling technique with contribution and allocation matrix. In the upcoming three parts, we take one quadrotor developed by our group as an instance to illustrate control designs. In Section $3, H_{\infty}$ loop-shaping and DOB design procedures are explained separately based on different perspectives to model uncertainty caused by load perturbation. Then the cross track based trajectory tracking algorithm is addressed in Section 4 . Finally, the paper compares robust performance between two strategies in greater detail and gives concluding remarks.

\section{A Universal Model}

We define MHeli as a class of UAV in which translational and rotational motions are just controlled by variation of each rotor speed, and the thrust force produced by the rotor is perpendicular to the airframe plane. The smallsized axial symmetry MHeli (AS-MHeli) is one of the most popular MHeli designs because of its symmetrical structure and economical feature to develop as a research platform. Additionally, most of the techniques which are studied based on AS-MHeli can be extended to various other designs of MHeli without loss of generality.

The generalized coordinates for the MHeli are [17]

$$
\mathbf{q}=(x, y, z, \phi, \theta, \psi)^{T},
$$

where $\xi=(x, y, z)^{T}$ denotes the position of the center of gravity (CoG) relative to one earth fixed frame (NED) and $\boldsymbol{\eta}=(\phi, \theta, \psi)^{T}$ contains the Euler angles that give the airframe orientation from body fixed frame to NED. Also as stated, $\lambda=(u, v, w)^{T}$ represents linear velocity of CoG in the body fixed frame and $\boldsymbol{\Omega}=(p, q, r)^{T}$ is rotation velocity in body fixed frame. By transforming body fixed frame to NED along with $(\psi, \theta, \phi), \mathbf{R} \dot{\xi}=\lambda, \widehat{\mathbf{R}} \dot{\boldsymbol{\eta}}=\boldsymbol{\Omega}$ could be concluded as follows:

$$
\begin{gathered}
\mathbf{R}=\left[\begin{array}{ccc}
\cos \theta \cos \psi & \cos \theta \sin \psi & -\sin \theta \\
\sin \theta \cos \psi \sin \phi-\sin \psi \cos \phi & \sin \theta \sin \psi \sin \phi+\cos \psi \cos \phi & \cos \theta \sin \phi \\
\sin \theta \cos \psi \cos \phi+\sin \psi \sin \phi & \sin \theta \sin \psi \cos \phi-\cos \psi \sin \phi & \cos \theta \cos \phi
\end{array}\right], \\
\widehat{\mathbf{R}}=\left[\begin{array}{ccc}
1 & 0 & -\sin \theta \\
0 & \cos \phi & \cos \theta \sin \phi \\
0 & -\sin \phi & \cos \theta \cos \phi
\end{array}\right] .
\end{gathered}
$$

Assuming that the airframe of MHeli is rigid, its general motion is divided into translational and rotational parts. Using the Newton-Euler formalism, the kinematics and dynamics of a rigid body under external forces applied to CoG can be expressed on earth fixed frame:

$$
m \ddot{\boldsymbol{\xi}}=\mathbf{R}^{T} \mathbf{F}+\mathbf{G}, \quad \mathbf{I} \dot{\boldsymbol{\Omega}}+\boldsymbol{\Omega} \times \mathbf{I} \boldsymbol{\Omega}=\boldsymbol{\tau},
$$




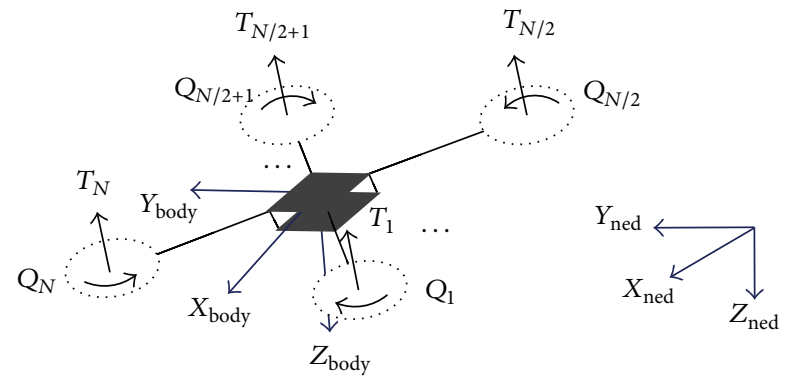

FIGURE 1: Free body diagram of AS-MHeli.

where I is inertia matrix, $\mathbf{G}$ denotes gravity expressed in NED, F represents the vector of nonconservative forces applied to the object, and $\boldsymbol{\tau}$ are external torque including aerodynamics effects and gyroscopic effects [18].

As AS-MHeli is powered by rotors, the differences of rotor speeds produce the roll motion, the pitch motion, and the yaw motion with various combinations. Note that the strategy we propose to define the subscript of each rotor facilitates modeling of dynamic equations (Figure 1).

In (3), the nonconservative forces external force $\mathbf{F}$ is combined with "lift force" $\mathbf{L}$ and drag force $\mathbf{D}$, both in body fixed frame, and the moments $\boldsymbol{\tau}$ are derived from aerodynamics and gyroscopic moments. Before giving $\mathbf{L}=$ $\left(L_{x}, L_{y}, L_{z}\right)^{T}$ and moment terms $\boldsymbol{\tau}=\left(\tau_{x}, \tau_{y}, \tau_{z}\right)^{T}$, we define a contribution matrix $\mathbf{E}_{6 \times N}$, its entry $e_{j i} \in\{-1,1\}, j=$ $1 \cdots 6, i=1 \cdots N$, and each entry represents the contribution of the $i$ th rotor to the $j$ th force or moments. Given that the thrust force produced by the rotor is vertical to airframe plane as defined above, $\mathbf{E}_{6 \times N}$ can be reduced to $\mathbf{E}_{4 \times N}$ because $\left(L_{x}, L_{y}\right)^{T}=(0,0)^{T}$. Then, $\mathbf{L}$ which is collection of thrust of rotors $T$ can be written as

$$
\mathbf{L}=\left(\begin{array}{c}
0 \\
0 \\
L_{z}
\end{array}\right)=\left(\begin{array}{c}
0 \\
0 \\
\sum e_{1 i} T_{i}
\end{array}\right), \quad i=1 \cdots N .
$$

For the general MHeli, gyroscopic moment is trivial in comparison with aerodynamics moment. Therefore, we neglect gyroscopic moment, retain the main features dominating dynamics characteristic, and give the generalized torque:

$$
\boldsymbol{\tau}=\left(\begin{array}{c}
\sum e_{2 i} \cdot d_{i} T_{i} \\
\sum e_{3 i} \cdot d_{i} T_{i} \\
\sum e_{4 i} \cdot Q_{i}
\end{array}\right), \quad i=1 \cdots N
$$

where $d$ is annotated as the distance from the rotors to CoG. As for drag force, which is mainly dependent on the velocity of the MHeli relative to ground, it can be yielded as

$$
\mathbf{D}=\left(\begin{array}{c}
K_{D x} \cdot\left(u-u_{w}\right)\left|u-u_{w}\right| \\
K_{D y} \cdot\left(v-v_{w}\right)\left|v-v_{w}\right| \\
K_{D z} \cdot\left(w-w_{w}\right)\left|w-w_{w}\right|
\end{array}\right),
$$

where $K_{D}$ is defined as an aerodynamic parameter involved in drag area and density of air and $\left(u_{w}, v_{w}, w_{w}\right)^{T}$ can be seen as wind velocity of CoG acting on body fixed frame.

Derived from our definition, the MHeli is controlled by the angular speeds of rotors $-\widetilde{\Omega}_{i}, i=1 \cdots N$, so the force and torque generated by each rotor can be modeled as

$$
T_{i}=K_{T} \widetilde{\Omega}_{i}^{2}, \quad Q_{i}=-C_{Q} \widetilde{\Omega}_{i}^{2}, \quad i=1 \cdots N
$$

where $K_{T}, C_{Q}$ are aerodynamic parameters involved in rotor and density of air. Taking the rotor and brushless motor as a whole, the angular speed of the rotor at a steady state would be linear with input of a brushless motor $U_{i}, i=1 \cdots N$, if the airframe is at hover state. However, if this angular speed change is accompanied by rotation or translation motion of the airframe, the dynamics of the rotor would be greatly different with the former one, where this phenomenon is known as load perturbation or rotor damping. Besides, its important roles in tracking the performance of a MHeli have been verified by both our theory analysis and realflight tests. Obviously, this characteristic of the rotor is a thorny question and is difficult to model, but it has an upper bound on magnitude dependant on frequency, so we studied this feature as a model unstructured uncertainty [2]. If we disregard load perturbation along with rotation and translation motion, the equation with transient response is as follows:

$$
\dot{\widetilde{\Omega}}_{i}+C_{\widetilde{\Omega}} \widetilde{\Omega}_{i}=K_{\widetilde{\Omega}} U_{i}, \quad i=1 \cdots N
$$

where $K_{\widetilde{\Omega}}, C_{\widetilde{\Omega}}$ are parameters involved in electromagnetic characteristics of motors.

Assume that the input is $\mathbf{u}=\left(u_{T}, u_{\phi}, u_{\theta}, u_{\psi}\right)^{T}$; that is, $\mathbf{U}=$ $\mathbf{D}_{s} \mathbf{u}$, where $\mathbf{U}=\left(U_{1}, U_{2} \cdots U_{N}\right)^{T}$ and $\mathbf{D}_{s}$ represents an $N \times 4$ allocation matrix determined by control strategy. As a matter of fact, $\mathbf{D}_{s}$ is essentially corresponding to $\mathbf{E}_{4 \times N}$, so it is notable that we can obtain various categories of AS-MHeli's model by simply changing $\mathbf{E}_{4 \times N}$. Consider

$$
\begin{gathered}
\mathbf{D}_{s}=\left(\begin{array}{cccc}
k_{11} e_{11} & k_{21} e_{21} & k_{31} e_{31} & k_{41} e_{41} \\
& \vdots & \vdots & \\
k_{1 i} e_{1 i} & k_{2 i} e_{2 i} & k_{3 i} e_{3 i} & k_{4 i} e_{4 i} \\
\vdots & \vdots & \\
k_{1 N} e_{1 N} & k_{2 N} e_{2 N} & k_{3 N} e_{3 N} & k_{4 N} e_{4 N}
\end{array}\right), \\
k_{j i} \in(0,1], \quad j=1 \cdots 4, i=1 \cdots N .
\end{gathered}
$$

To better apply the universal model and illustrate control strategy, we extract a quadrotor, controlled by 4 rotors, model on the basis of self-designed platform (Figure 2). As to our quadrotor platform, $\mathbf{E}_{4 \times N}$ and $k$ are designed to make the best use of symmetry frame and guarantee decoupling between different channels to a large extent, thereby simplifying model and control strategy. 


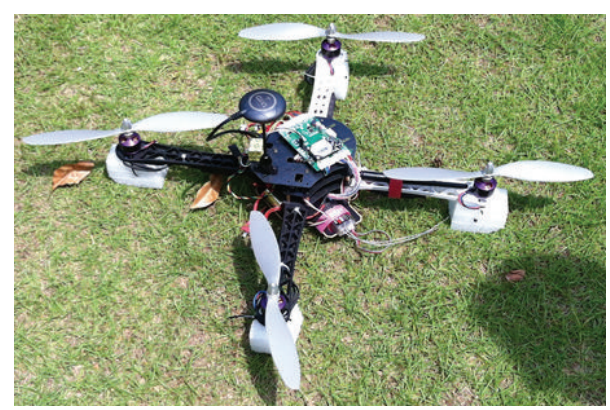

FIGURE 2: Quadrotor platform.

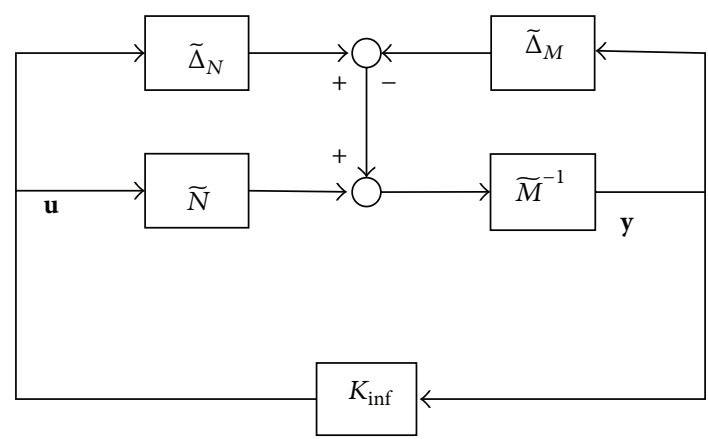

Figure 3: Standard presentation of $H_{\infty}$ control problem.

\section{Attitude Tacking}

The motion of MHeli is dependent on aerodynamic force generated by the angular speeds of four rotors [19], yet the numerical model of the load perturbation or rotor damping is indeterminate and difficult to express clearly. Undoubtedly, an exquisite dynamic model of rotor would greatly benefit a lot of researches on a multirotor UAV, but it is arduous and formidable to identify a perfect model. In this paper, we are devoted to proposing valuable methods in both theory and practice from two different views to solve this model uncertainty problem.

3.1. $H_{\infty}$ Loop-Shaping. The classic formulation of robust control problem is primarily presented in [20], where $w$ is the so-called external disturbance [21]. In particular, the control input $\mathbf{u}=\left(U_{T}, U_{\phi}, U_{\theta}, U_{\psi}\right)^{T}$, the controlled outputs $z=$ $(\phi, \theta, \psi)^{T}$, and the measured variables $\mathbf{y}=(\phi, \theta, \psi, p, q, r)^{T}$ as to our MHeli system. In this section, the uncertainty is modeled as perturbations on the normalized coprime factors of the nominal plant [9] (see Figure 3).

Where $\widetilde{M}$ derives from left coprime factorization $G=$ $\widetilde{M}^{-1} \widetilde{N}$, this approach could give a general framework for representing the unknown uncertainties $\widetilde{\Delta}_{N}, \widetilde{\Delta}_{M}$, as long as uncertainty is stable and norm-bounded, which is satisfied by its upper-bound nature. Then the perturbed system is robustly stable if only [22]

$$
\left\|\left(\begin{array}{c}
I \\
K_{\text {inf }}
\end{array}\right)\left(I-G K_{\text {inf }}\right)^{-1} \widetilde{M}^{-1}\right\|_{\infty} \leq \gamma=\frac{1}{\varepsilon} .
$$

Generally, before designing the feedback controller $K_{\text {inf }}$, the nominal plant $G$ is augmented by the pre- and postcompensators $\left(W_{1}\right.$ and $\left.W_{2}\right)$ to give a desired shape to improve open-loop frequency response. Thus, $G$ in (11) is replaced by $G_{s}=W_{1} G W_{2}$. The weight functions could be designed by shaping the nominal plant singular values with two principles: $W_{1}$ should provide sharp decline at low frequency; otherwise, $W_{2}$ should bring extra gain at high frequency to attenuate noise. In this case, if the shaped system obtains stability and performance, it would be identical to the original system.

Model reduction is then performed on $K_{\text {inf }}$ to get a 10state system. Figure 4 with frequency response states that the performance of the resulting controller is relatively unaffected over most frequencies. Following this, we notice that the resulting state space controller is effectively sparse, as many entries are very close to zero. This superiority is beneficial from the elaborate selection of contribution and allocation matrix, which guarantees decoupling between different channels to a large extent.

As is well known, the closed-loop properties of a controlled system can be valued by sensitivity function and complementary sensitivity function:

$$
S=\frac{1}{I+G_{s} K_{\mathrm{inf}}}, \quad T=\frac{G_{s} K_{\mathrm{inf}}}{I+G_{s} K_{\mathrm{inf}}} .
$$

In particular, we introduce $F=\gamma T / W_{2}$ to testify how the poles of $W_{1}$ affect the property of loop-shaped system [2].

Generally, for good performance, we require $\bar{\sigma}(S)$ to be small (at low frequency) and for good robust stability properties we require $\bar{\sigma}(T)$ to be small (at high frequency) [23]. The rough robust performance and stability are displayed in Figure 5. The controller can now tolerate almost $10 \mathrm{~dB}$ uncertainty at $10 \mathrm{rad} / \mathrm{s}$ and also provide $-160 \mathrm{~dB}$ sensitivity at low frequencies. Additionally, the suggested stability margin is satisfactory. On the other hand, $F$ shows that the poles of $W_{1}$ make the match in $T$ quite good, which means that $W_{1}$ really improve performance of the original plant.

3.2. Disturbance Observer Based Linear Quadratic Gaussian (LQG) Controller. Unlike the above traditional robust control algorithm, which integrates robust margin requirements into the design process and sacrifices tracking performance to cater to robust stability, this section proposes taking model uncertainty as external disturbance instead of internal perturbation and introduces the two-degree-of-freedom structure [24], which enables us to design optimal controller and DOB separately, to satisfy desired performance specifications and robust margin requirements at the same time.

The linearized multivariable system can be rewritten as $\dot{\mathbf{x}}=\mathbf{A x}+\mathbf{B u}, \mathbf{y}=\mathbf{C x}$, where $\mathbf{x}=\left(\phi, \theta, \psi, p, q, r, \widetilde{\Omega}_{1}\right.$, $\left.\widetilde{\Omega}_{2}, \widetilde{\Omega}_{3}, \widetilde{\Omega}_{4}\right)^{T}, \mathbf{y}=(\phi, \theta, \psi, p, q, r)^{T}$.

Linear quadratic regulation (LQR) is an optimal control with cost function $J=\int\left(\mathbf{x}^{\mathrm{T}} \mathbf{Q} \mathbf{x}+\mathbf{u}^{\mathrm{T}} \mathbf{R} \mathbf{u}\right) d t$. It automatically provides some upper bounds on the magnitude of the sensitivity function and its complementary [25]. However, these bounds are frequency-independent and may not result in appropriate loop-shaping, so a feed-forward compensation 

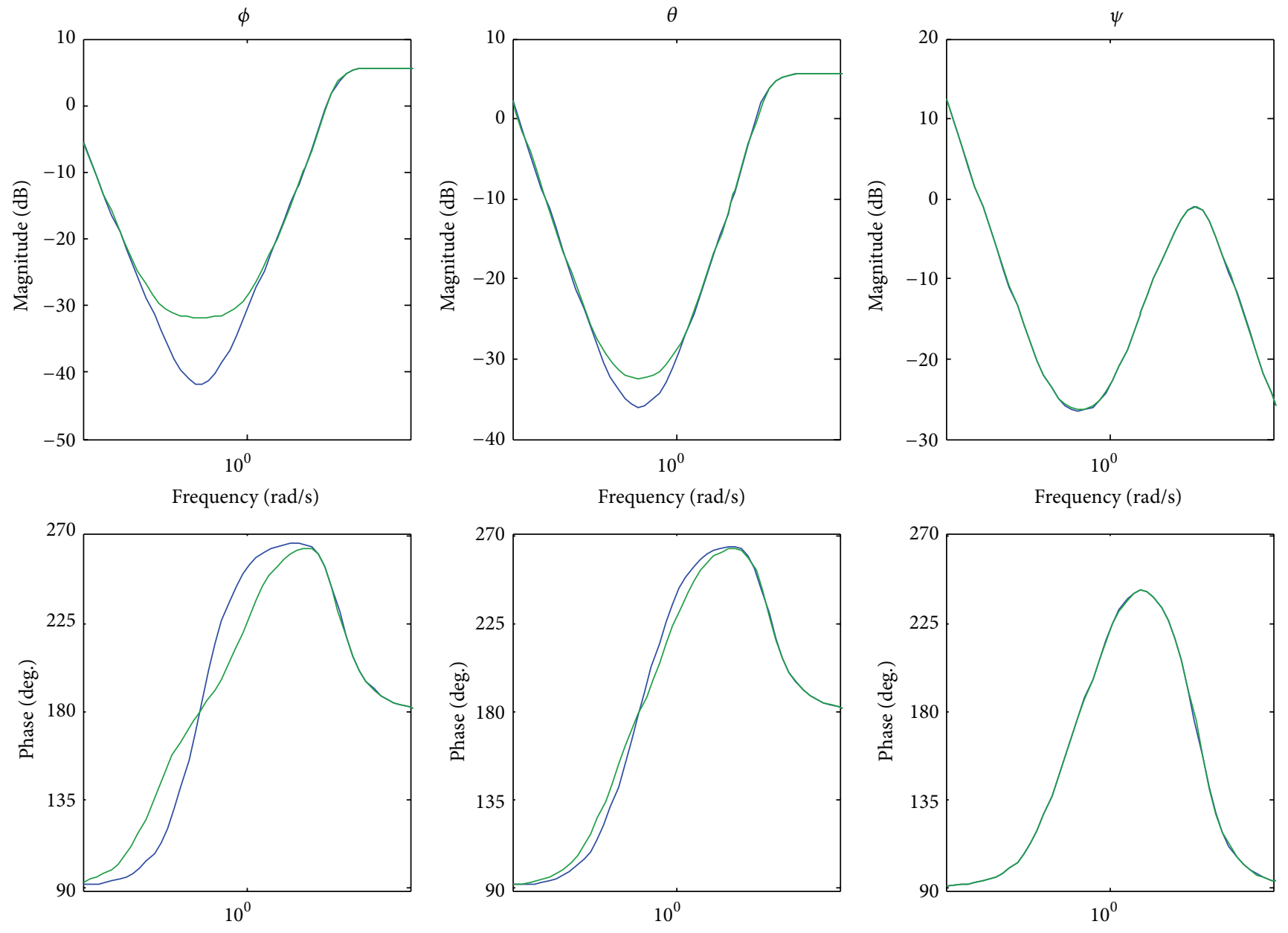

Frequency $(\mathrm{rad} / \mathrm{s})$

Frequency $(\mathrm{rad} / \mathrm{s})$

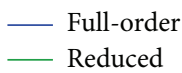

- Full-order

Frequency $(\mathrm{rad} / \mathrm{s})$

- Reduced

— Full-order
- Reduced

FIgURE 4: Comparison with the original and deduced $H_{\infty}$ controller.

variable $\mathbf{K}_{\mathbf{g}} \mathbf{x}_{\text {ref }}$ is designed to guarantee competing tracking for the original outputs of interest.

In addition, as for our quadrotor platform, the angular speed of rotors $\left(\widetilde{\Omega}_{1}, \widetilde{\Omega}_{2}, \widetilde{\Omega}_{3}, \widetilde{\Omega}_{4}\right)^{T}$ is unobservable, so an observer is introduced to estimate those four states. Then, an output-feedback optimal controller which combines LQR and observer is given by

$$
\dot{\hat{\mathbf{x}}}=(\mathbf{A}-\mathbf{L C}) \widehat{\mathbf{x}}+\mathbf{B u}+\mathbf{L y}, \quad \mathbf{u}=-\mathbf{K}_{\mathbf{c}} \widehat{\mathbf{x}}+\mathbf{K}_{\mathbf{g}} \mathbf{y}_{\text {ref }},
$$

where $\mathbf{K}_{\mathbf{c}}$ and $\mathbf{L}$ denote LQR controller and state estimator, respectively, and $\mathbf{K}_{\mathbf{g}}$ is the so-called feed-forward compensator. Noticeably, we employ the Kalman filter as our observer for its great robustness to white Gaussian noise, and this output-feedback optimal controller is usually known as LQG controller (see Figure 6).

Generally, great reference tracking can be achieved by designing a LQG controller with small complementary sensitivity function [5]; however, a LQG controller only has mediocre performance on disturbance rejection.
The capability of robustness to external disturbance would be compensated by DOB, which is widely used to estimate and suppress exotic disturbance [26].

Figure 7 is a classic DOB structure for a SISO system, which consists of a nominal model $G_{n}$ and a low-pass filter $Q_{n}$; thus, selection of $G_{n}$ and design of $Q_{n}$ are key issues of DOB configuration. In this paper, we take the roll channel as an instance to present our design idea.

We obtained the nominal model of roll channel $P_{n}$ by linearizing original nonlinear model at the state when the system is vulnerable to this kind of disturbance, namely, as the aircraft rotates at a constant angular speed. On the other hand, the low-pass filter $Q_{n}$ has the same or higher order with $P_{n}$ and meets the requirements that external disturbance and measurement noise can be attenuated simultaneously in their separated frequency ranges. If $Q_{n}$ has higher bandwidth than low-frequency disturbance and lower cutoff frequency than high-frequency measurement noise, disturbance is attenuated and the mismatch of nominal model is compensated and high-frequency measurement noise rejection is achieved. 


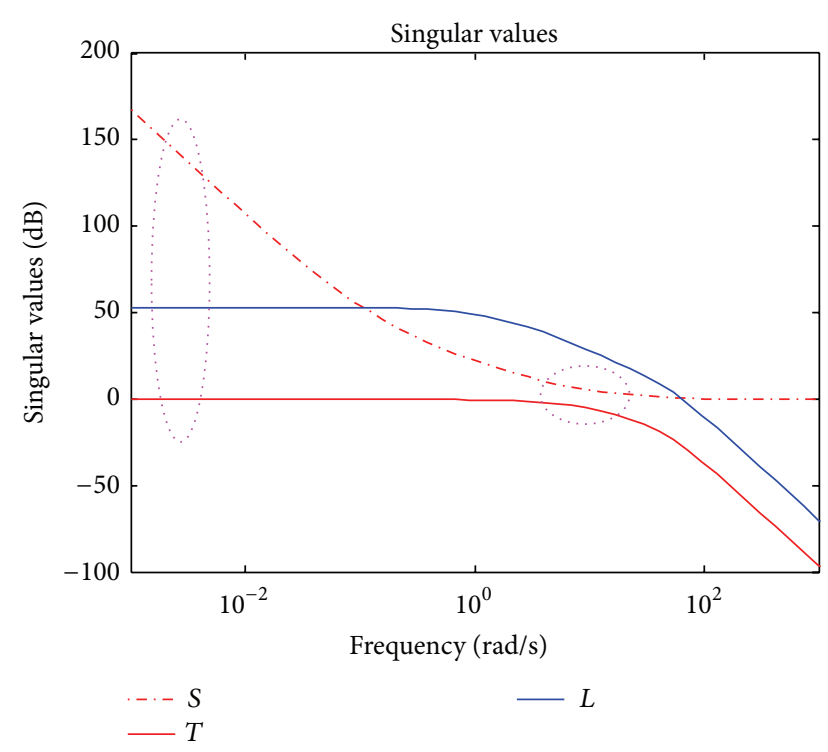

FIGURE 5: Robust stability margin.

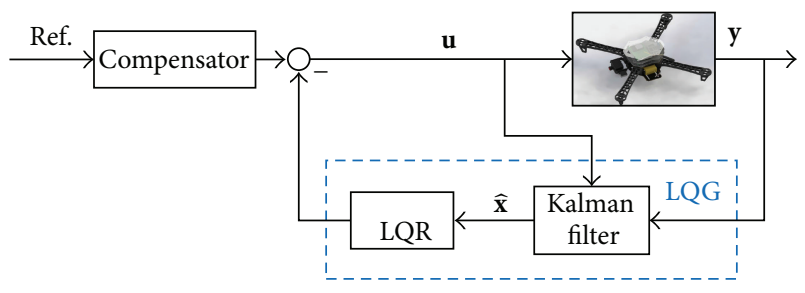

FIGURE 6: LQG controller.

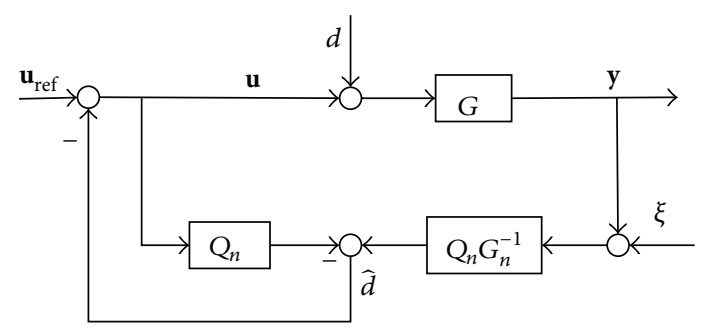

Figure 7: Classic DOB structure.

In order to meet the above requirements, the paper suggests that the binomial $Q_{n}$-filter should have the following form:

$$
Q_{n}(s)=\frac{\sum_{k=0}^{m-n}(1+\tau s)^{k}}{\prod_{k=n}^{m}(1+\tau s)^{k}}
$$

where $m$ is the order of $Q_{n}, n$ is the relative degree of $G_{n}$, and $\tau=1 / \omega_{c}$ denotes time constant of $Q_{n}$. Although the tracking performance could be enhanced by increasing the order and bandwidth of $Q_{n}$, excessive order and bandwidth of $Q_{n}$ may lead to destruction of the stability [27]. Therefore, it is preferred that $m$ is no more than $n+3$ and $\omega_{c}$ is less than twice of cutoff frequency of $G_{n}$. Besides, to compensate

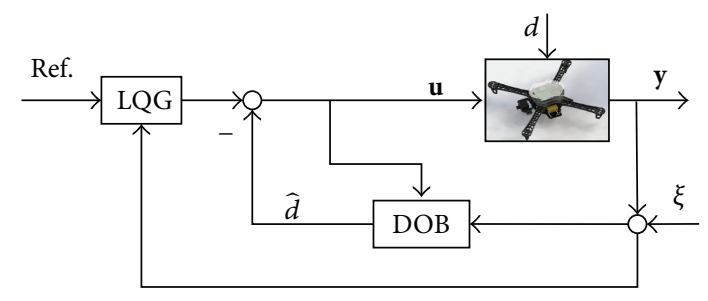

FIGURE 8: DOB-based LQG controller.
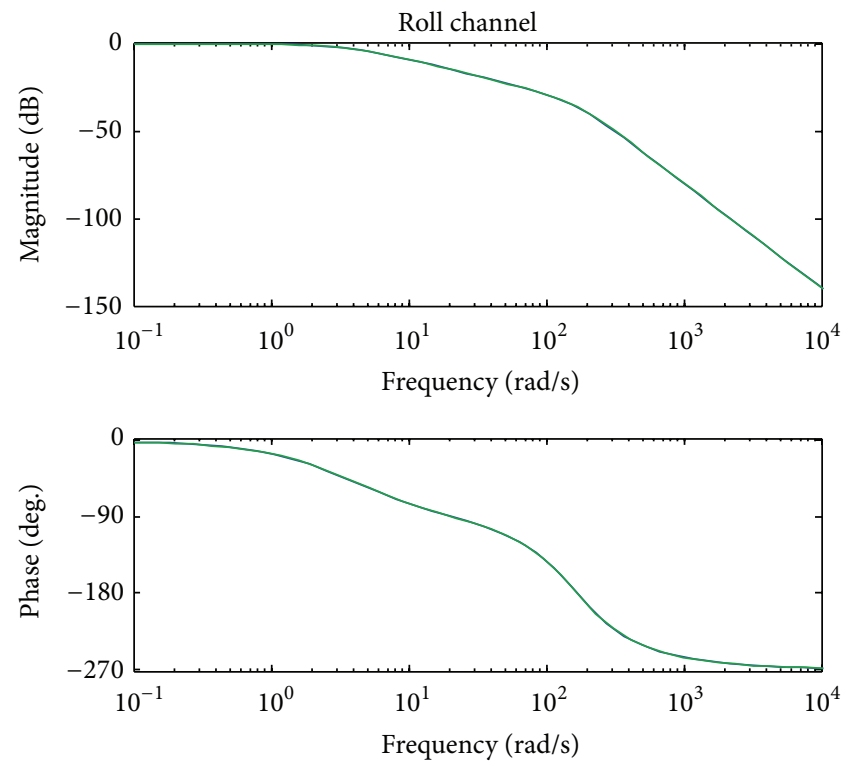

Without DOB
With DOB

Figure 9: Performance comparison.

phase lag accompanied with observer, a lead-compensator is directly cascaded ahead of $Q_{n}$ :

$$
W_{n}(s)=\frac{a \cdot \tau s+1}{b \cdot \tau s+1},
$$

where $a$ and $b$ are parameters required to be regulated according to design of $Q_{n}$. So far, DOB design for the roll channel is completed, and designs for another two channels can be employed under the guidance of the same idea.

Before combining the optimal controller and disturbance observer, we have to transform LQG into unity-feedback formation. Then, the DOB can be integrated in the light of two-freedom theory and its estimation is added to control input as the disturbance cancellation part (Figure 8).

Note that stability of the DOB-integrated system is not ensured by stability conditions of the LQG-controlled system because the nominal external disturbance is closely related to system states, which may induce smaller stability margin by introducing DOB. Taking the roll channel as the example as before, Figure 9 illustrates that stability margin does not decrease with $Q_{n}$ and other compensators, and dynamic 


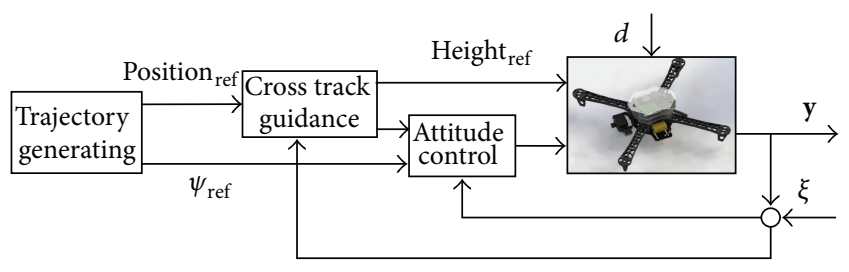

FIGURE 10: Trajectory tracking strategy.

response of the system is not implicated along a large range of frequencies. Specifically, phase lag induced by an observer is eliminated for the introduction of an appropriate lead-compensator.

\section{Cross Track Based Trajectory Tracking}

To validate the robustness performance on trajectory tracking, this section realizes the guidance law and trajectory generator.

When ensuring that the objective converges to the reference trajectory, we consider the design of a cross track based guidance system. The cross track control refers to the process of forcing the aircraft to track a given trajectory by minimizing the normal distance to the path, namely, the cross track error [28]. Cross track based guidance has the ability to minimize trajectory errors while moving between waypoints, even enabling the helicopter not to leave a commanded altitude which may occur when abruptly changing the attitude reference with huge command values [29]. It benefits tracking performance without sacrificing MHelis' agile maneuvering.

Benefitting from the careful selection of contribution and allocation matrix, our quadrotor model achieves the large degree of decoupling between height control and attitude control. Given the aforementioned characteristic, the paper proposes the trajectory tracking structure (see Figure 10).

Derived from information on position and yaw, the cross track errors $d_{x}, d_{y}, d_{z}$ which would be used in guidance law can be yielded:

$$
\begin{aligned}
& d_{x}=z_{\text {loss }}(\Delta x \cos (\Delta \psi)+\Delta y \sin (\Delta \psi)), \\
& d_{y}=z_{\text {loss }}(\Delta x \sin (\Delta \psi)+\Delta y \cos (\Delta \psi)), \\
& d_{z}=\frac{\Delta z}{(\cos \phi \cos \theta)},
\end{aligned}
$$

where $\Delta x, \Delta y, \Delta z$, and $\Delta \psi$ are deviation between measurements and reference and $z_{\text {loss }}$ is a constant to antagonize the height loss problem [17]. An adapted algorithm with tuning parameters $a, b$, and $c$ is designed on the basis of cross track error:

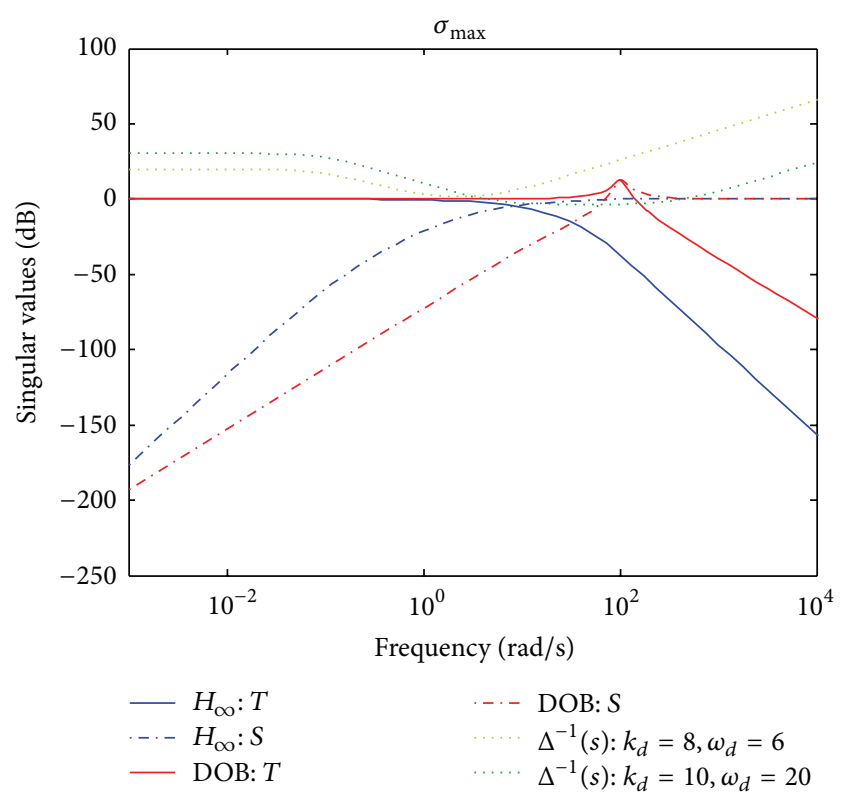

FIGURE 11: Robust stability and tracking performance.

$$
\begin{aligned}
& \left(\begin{array}{c}
u_{T} \\
\bar{u}_{\phi} \\
\bar{u}_{\theta}
\end{array}\right) \\
& =\mathbf{R}\left(\begin{array}{c}
-a_{T} d_{z}-b_{T} \int d_{z} \\
-a_{\phi} \max \left(k_{\Delta y} \cdot \Delta y, d_{y}\right)-b_{\phi} \int \max \left(k_{\Delta y} \cdot \Delta y, d_{y}\right) \\
-a_{\theta} \max \left(k_{\Delta x} \cdot \Delta y, d_{x}\right)-b_{\theta} \int \max \left(k_{\Delta x} \cdot \Delta y, d_{x}\right)
\end{array}\right) \\
& +\mathbf{R}^{\mathrm{T}}\left(\begin{array}{c}
-c_{T} w \\
-c_{\phi} v \\
-c_{\theta} u
\end{array}\right),
\end{aligned}
$$

where $\bar{u}_{\phi}, \bar{u}_{\theta}$ are corresponding with reference of attitude controller and $u_{T}$ is delivered to model forthrightly. As for $u_{\psi}$, it is generated by attitude controller rather than guidance law.

\section{Simulation Results and Robust Analysis}

As mentioned above, rotor damping uncertainty $\Delta$ is a class of model unstructured uncertainties with an upperbound magnitude dependant on frequency, so its upper-limit inequality is expressed as

$$
|\Delta(j \omega)| \leq\left|V_{\mathrm{hf}}(j \omega)\right| .
$$

Then designs with a feedback system are robustly stable against uncertainty if $\bar{\sigma}(\Delta(j \omega) \cdot T(j \omega))<1$; that is,

$$
\left\|V_{\mathrm{hf}}(j \omega) T(j \omega)\right\|_{\infty}<1 \text {, }
$$


Position error: compare $H_{\infty}$ with DOB
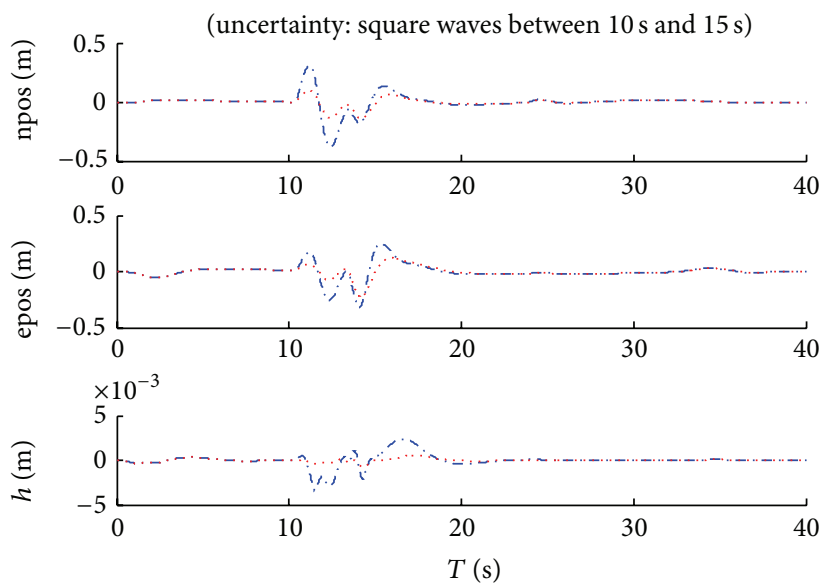

$--H_{\infty}$

DOB

(a)
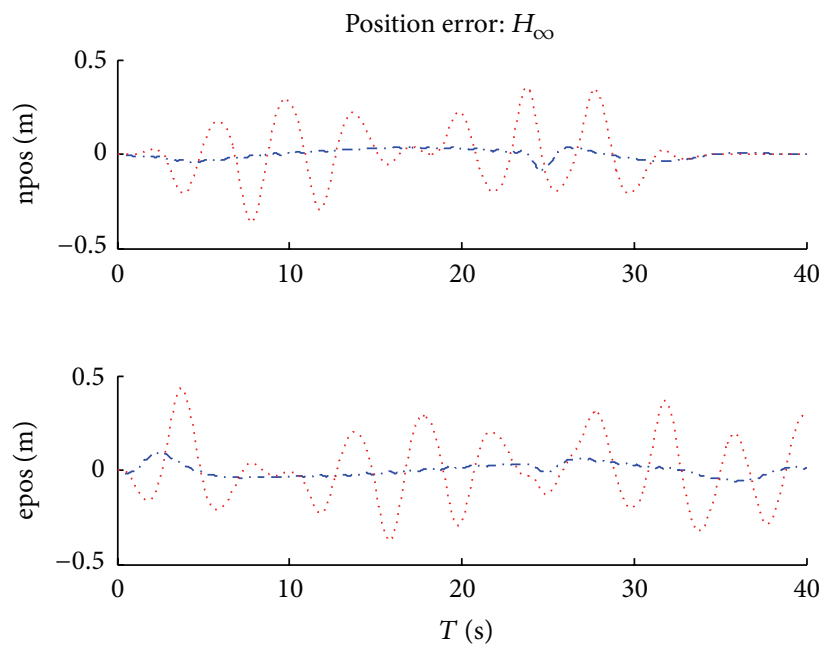

$\cdots k_{d}=8, \omega_{d}=12$

(c)
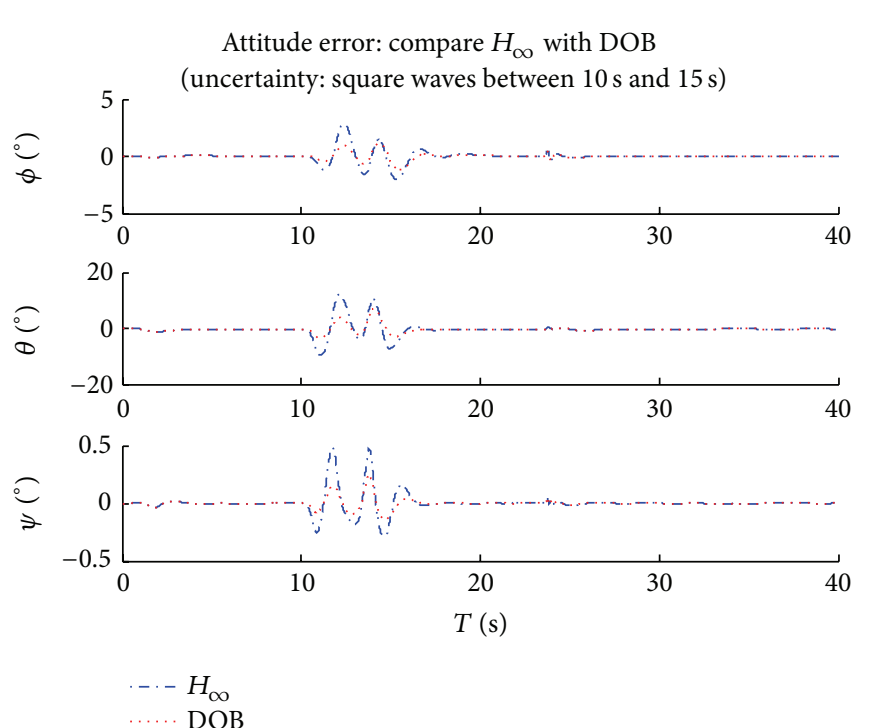

(b)
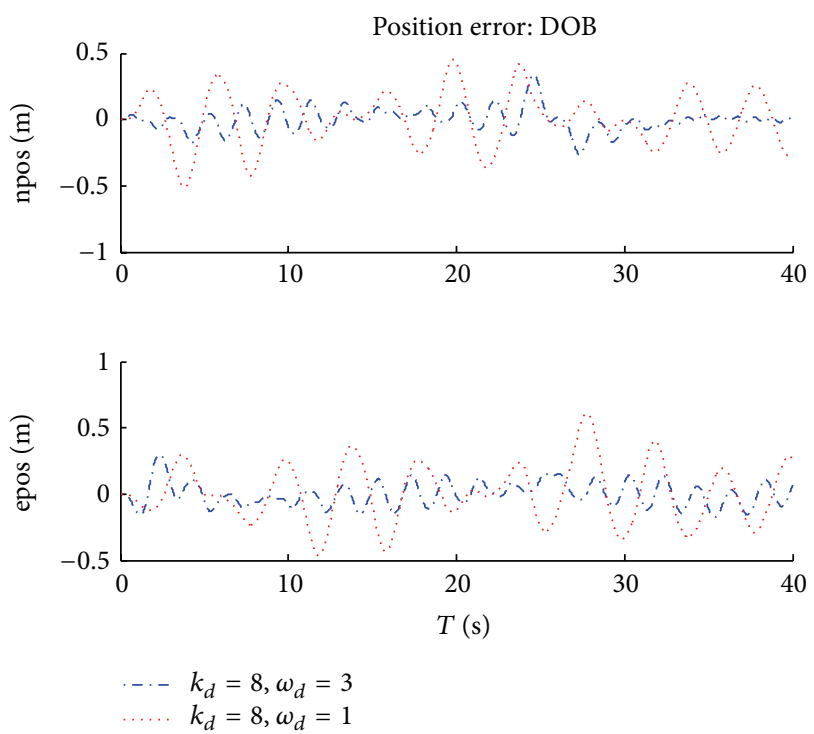

(d)

FIGURE 12: Trajectory tracking with model uncertainty.

where $T$ is the complementary sensitivity function. On the other hand, the robust tracking performance is dependent on singular value at low frequency, so the feedback control loop should be designed by

$$
\min \left\|\rho \cdot V_{\mathrm{lf}}(s) S(s)\right\|_{\infty}<1,
$$

where $S$ is the sensitivity function and $\rho \in(0,1)$. Thus, for meeting the requirements of robust stability and tracking performance simultaneously, the final optimal system should satisfy [17]

$$
\min \left\|\left(\begin{array}{c}
V_{\mathrm{hf}}(s) T(s) \\
\rho \cdot V_{\mathrm{lf}}(s) S(s)
\end{array}\right)\right\|_{\infty}<1
$$

According to dynamic characteristics of a MHeli, rotor damping uncertainty of rotor interferes with the rotation and translation motion of aircraft frame and rotation motion indirectly influences translation motion, so we propose integrating model uncertainty into rotation motion equations: $\dot{\boldsymbol{\Omega}}=[f(\boldsymbol{\Omega}, \boldsymbol{\omega})+g(\boldsymbol{\omega}) \mathbf{u}]+\Delta$. As load perturbation typically has relatively low frequency, it is natural that the criterion emphasizes the behavior at low frequency. Finally, we select cosine function $\Delta=k_{d} \sin \left(\pi \omega_{d} t+1\right)$ for its abundant information and variable magnitude and frequency.

With the analysis of $\bar{\sigma}(T)$ and $\bar{\sigma}(S)$, Figure 11 compares the robust performance obtained from $\mathrm{H}_{\infty^{-}}$and DOBbased tracking controller. The frequency response of $\bar{\sigma}(T)$ illustrates that the closed loop with $H_{\infty}$ shape obtains 
a larger robust stability margin, approximately $k_{d} \leq 10$, $\omega_{d} \leq 20$, especially getting better performance under higher bandwidth uncertainty, compared with approximately $k_{d} \leq$ $8, \omega_{d} \leq 6$ for a DOB-based controller. On the contrary, the computed sensitivity $S$ of a DOB-based system displays a steeper decline at low frequency, suggesting better robust tracking performance. This may result from less tradeoff constraint of attitude tracking design with two-degree-offreedom structure.

To test and analyze tracking performance of two robust strategies under rotor damping uncertainty, we designed many simulation experiments and selected the most representative ones, which command quadrotors to track a circle with $4 \mathrm{~m} / \mathrm{s}$ forward velocity (Figure 12). During the tracking, the heading of the aircraft is in accord with tangent of reference trajectory all along, and the aircraft is required to start and end the tracking at the same point with hover state.

Figure 12 illustrates tracking performance of two controllers when the system is subjected to rotor damping uncertainty simulated by different sorts of forms. In Figures 12(a) and 12(b), unexpected uncertainty occurring between $10 \mathrm{~s}$ and $15 \mathrm{~s}$ is composed of two square waves, which have opposite sign and the same magnitude up to 6. Otherwise, Figures 12(c) and 12(d) note uncertainty continuously accompanied with motion of airframe, and this uncertainty is simulated by cosine function $k_{d} \sin \left(\pi \omega_{d} t+1\right)$ with variable magnitudes and frequencies.

The results demonstrate that the quadrotor is able to track reference under transitory load perturbation and continuous model uncertainty, and the system reaches satisfactory robust performance on trajectory tracking and fairly aggressive velocity reference tracking. As rotor damping uncertainty accompanies motion of airframe, $\psi$ is less affected by this uncertainty. Figure 12(b) shows that both two techniques can attenuate sporadic uncertainty with relatively large magnitude. Figures 12(c) and 12(d) conclude that a DOB-based controller reaches better rejection performance, though an $H_{\infty}$-based controller acquires larger stability margin. These conclusions are compatible with those resulting from the above analytic method. It is notable that the simulation responses in Figures 12(c) and 12(d) show that the two methods both handle high-frequency uncertainties better.

In addition to the above conclusion, some notes are worth highlighting: (1) $H_{\infty}$ loop-shaping based on MIMO design method may enhance coupling between attitude channels; (2) the shaped system cannot reflect original features of plant, so it is difficult to reach a satisfactory balance.

\section{Conclusions}

As for a multirotor helicopter, its dynamics model could be only implicated to a contribution matrix by employing the universal model. Besides, simplified model and decoupling characteristics can be obtained with delicate selection of allocation matrices. As rotor damping uncertainty may threaten system stability and cause deterioration of tracking performance, it is important to guarantee rejection for pursuing excellent maneuverability of a MHeli. In comparison with other robust control methods, $H_{\infty}$ loop-shaping is a convenient way to trade off the robustness according to uncertainty bound and reach a quite large stability margin. However, it may offset tracking performance when chasing large robust stability margin. On the other hand, though with relatively small stability margin, a DOB-based control structure can resolve these conflicting specifications by virtue of the flexibility of two-degree-of-freedom structure. It is also noted that the control methodology addressed in this paper can be easily extended to other kinds of multiplerotor helicopters and implemented in practice even with an embedded system.

\section{Conflict of Interests}

The authors declare that there is no conflict of interests regarding the publication of this paper.

\section{References}

[1] G. M. Hoffmann, H. Huang, S. L. Waslander, and C. J. Tomlin, "Quadrotor helicopter flight dynamics and control: theory and experiment," in Proceedings of the AIAA Guidance, Navigation and Control Conference and Exhibit, vol. 2, pp.1670-1689, Hilton Head, SC, USA, August 2007.

[2] K. Glober and D. McFarlane, "Robust stabilization of normalized coprime factors: an explicit $\mathrm{H}_{\infty}$ solution," in Proceedings of the American Control Conference, Atlanta, Ga, USA, 1988.

[3] G. V. Raffo, M. G. Ortega, and F. R. Rubio, "An integral predictive/nonlinear $H_{\infty}$ control structure for a quadrotor helicopter," Automatica, vol. 46, no. 1, pp. 29-39, 2010.

[4] T. Lee, M. Leok, and N. H. Mcclamroch, "Nonlinear robust tracking control of a quadrotor UAV on SE(3)," Asian Journal of Control, vol. 15, no. 2, pp. 391-408, 2013.

[5] O. Bouhali and H. Boudjedir, "Neural network control with neuro-sliding mode observer applied to quadrotor helicopter," in Proceedings of the International Symposium on Innovations in Intelligent Systems and Applications (INISTA '11), pp. 24-28, IEEE, June 2011.

[6] A. Das, K. Subbarao, and F. Lewis, "Dynamic inversion with zero-dynamics stabilisation for quadrotor control," IET Control Theory and Applications, vol. 3, no. 3, pp. 303-314, 2009.

[7] G. M. Hoffmann, H. Huang, S. L. Waslander, and C. J. Tomlin, "Quadrotor helicopter flight dynamics and control: theory and experiment," in Proceedings of the AIAA Guidance, Navigation, and Control Conference, vol. 2, pp. 1670-1689, August 2007.

[8] B. Zhao, B. Xian, Y. Zhang, and X. Zhang, "Immersion and invariance based adaptive attitude tracking control of a quadrotor UAV in the presence of parametric uncertainty," in Proceedings of the 33rd Chinese Control Conference (CCC '14), pp. 1932 1937, July 2014.

[9] B. J. Bialy, J. Klotz, K. Brink, and W. E. Dixon, "Lyapunov-based robust adaptive control of a quadrotor UAV in the presence of modeling uncertainties," in Proceedings of the 1st American Control Conference (ACC '13), pp. 13-18, Washington, DC , USA, June 2013.

[10] Z. T. Dydek, A. M. Annaswamy, and E. Lavretsky, "Adaptive control of quadrotor UAVs: a design trade study with flight evaluations," IEEE Transactions on Control Systems Technology, vol. 21, no. 4, pp. 1400-1406, 2013. 
[11] I. J. Gabe, V. F. Montagner, and H. Pinheiro, "Design and implementation of a robust current controller for VSI connected to the grid through an LCL filter," IEEE Transactions on Power Electronics, vol. 24, no. 6, pp. 1444-1452, 2009.

[12] C.-S. Liu and H. Peng, "Disturbance observer based tracking control," Transactions of the ASME-Journal of Dynamic Systems, Measurement and Control, vol. 122, no. 2, pp. 332-335, 2000.

[13] K. Lee, J. Back, and I. Choy, "Disturbance observer based trajectory tracking controller for quadrotor," in Proceedings of the 12th International Conference on Control, Automation and Systems (ICCAS '12), pp. 157-161, IEEE, October 2012.

[14] F. Chen, F. Lu, B. Jiang, and G. Tao, "Adaptive compensation control of the quadrotor helicopter using quantum information technology and disturbance observer," Journal of the Franklin Institute, vol. 351, no. 1, pp. 442-455, 2014.

[15] A. Salihbegovic, E. Sokic, N. Osmic, and M. Hebibovic, "High performance disturbance observer based control of the nonlinear 2DOF helicopter system," in Proceedings of the 24th International Conference on Information, Communication and Automation Technologies (ICAT '13), pp. 1-7, IEEE, November 2013.

[16] A. Salihbegovic, E. Sokic, N. Osmic, and M. Hebibovic, "High performance disturbance observer based control of the nonlinear 2DOF helicopter system," in Proceedings of the 24th International Conference on Information, Communication and Automation Technologies (ICAT '13), bih, November 2013.

[17] K. Alexis, C. Papachristos, G. Nikolakopoulos, and A. Tzes, "Model predictive quadrotor indoor position control," in Proceedings of the 19th Mediterranean Conference on Control \& Automation (MED '11), pp. 1247-1252, IEEE, Corfu, Greece, June 2011.

[18] L. R. G. Carrillo, A. E. Dzul López, R. Lozano, and C. Pégard, "Modeling the quad-rotor mini-rotorcraft," in Quad Rotorcraft Control, Advances in Industrial Control, pp. 23-34, Springer, London, UK, 2013.

[19] P.-J. Bristeau, P. Martin, E. Salaün, and N. Petit, "The role of propeller aerodynamics in the model of a quadrotor UAV," in Proceedings of the European Control Conference, Budapest, Hungary, August 2009.

[20] K. Zhou, J. C. Doyle, and K. Glover, Robust and Optimal Control, Prentice Hall, New Jersey, NJ, USA, 1996.

[21] K. Zhou, J. C. Doyle, and K. Glover, Robust and Optimal Control, Prentice Hall, Upper Saddle River, NJ, USA, 1996.

[22] M. H. Sadraey, Design of a nonlinear robust controller for a complete unmanned aerial vehicle mission [Ph.D. thesis], University of Kansas, Lawrence, Kan, USA, 2006.

[23] D. McFarlane and K. Glover, "A loop-shaping design procedure using $H_{\infty}$ synthesis," IEEE Transactions on Automatic Control, vol. 37, no. 6, pp. 759-769, 1992.

[24] T. Umeno and Y. Hori, "Robust speed control of DC servomotors using modern two degrees-of-freedom controller design," IEEE Transactions on Industrial Electronics, vol. 38, no. 5, pp. 363-368, 1991.

[25] J. P. Hespanha, Lecture Notes on $L Q R / L Q G$ Controller Design, University of California, 2005.

[26] J. N. Yun, J. Su, Y. I. Kim, and Y. C. Kim, "Robust disturbance observer for two-inertia system," IEEE Transactions on Industrial Electronics, vol. 60, no. 7, pp. 2700-2710, 2013.

[27] J. R. Ryoo, T.-Y. Doh, and M. J. Chung, "Robust disturbance observer for the track-following control system of an optical disk drive," Control Engineering Practice, vol. 12, no. 5, pp. 577585, 2004.

[28] E. Børhaug, K. Y. Pettersen, and A. Pavlov, "An optimal guidance scheme for cross-track control of underactuated underwater vehicles," in Proceedings of the 14th Mediterranean Conference on Control and Automation (MED '06), June 2006.

[29] T. Puls, M. Kemper, R. Küke, and A. Hein, "GPS-based position control and waypoint navigation system for quadrocopters," in Proceedings of the IEEE/RSJ International Conference on Intelligent Robots and Systems (IROS '09), pp. 3374-3379, October 2009. 

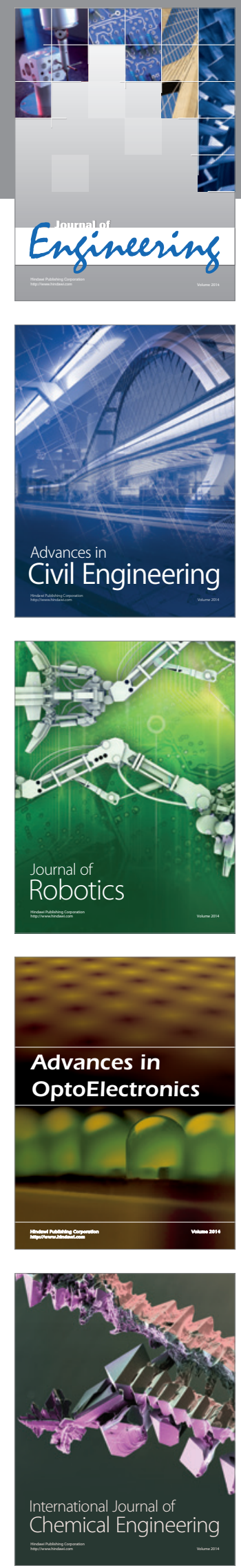

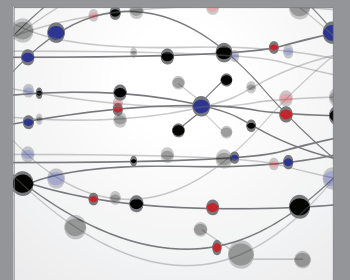

The Scientific World Journal
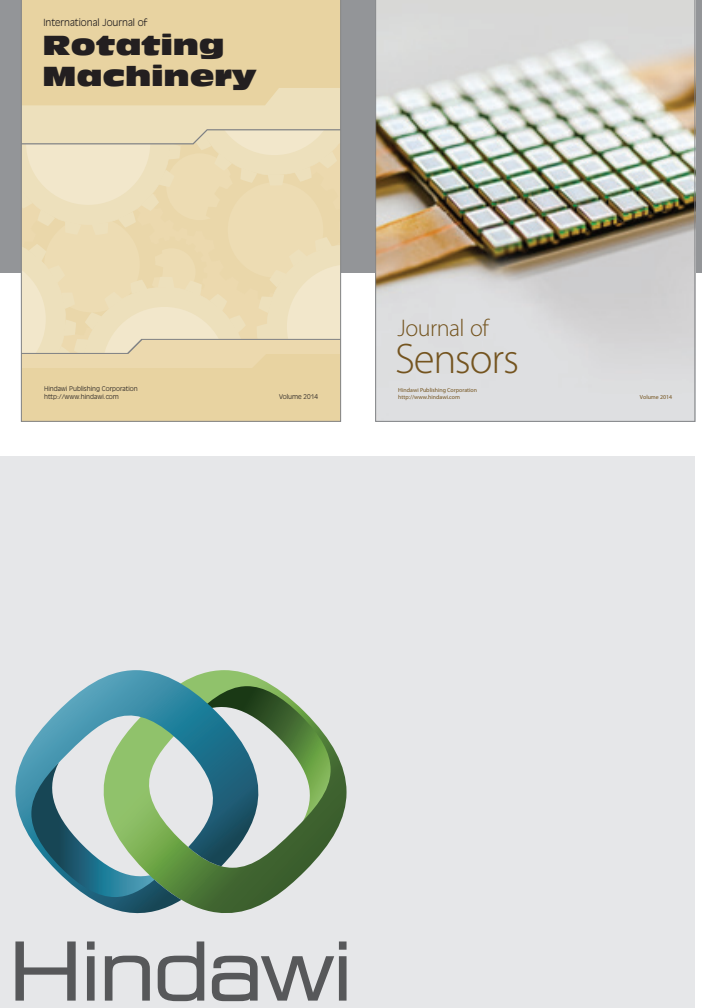

Submit your manuscripts at http://www.hindawi.com
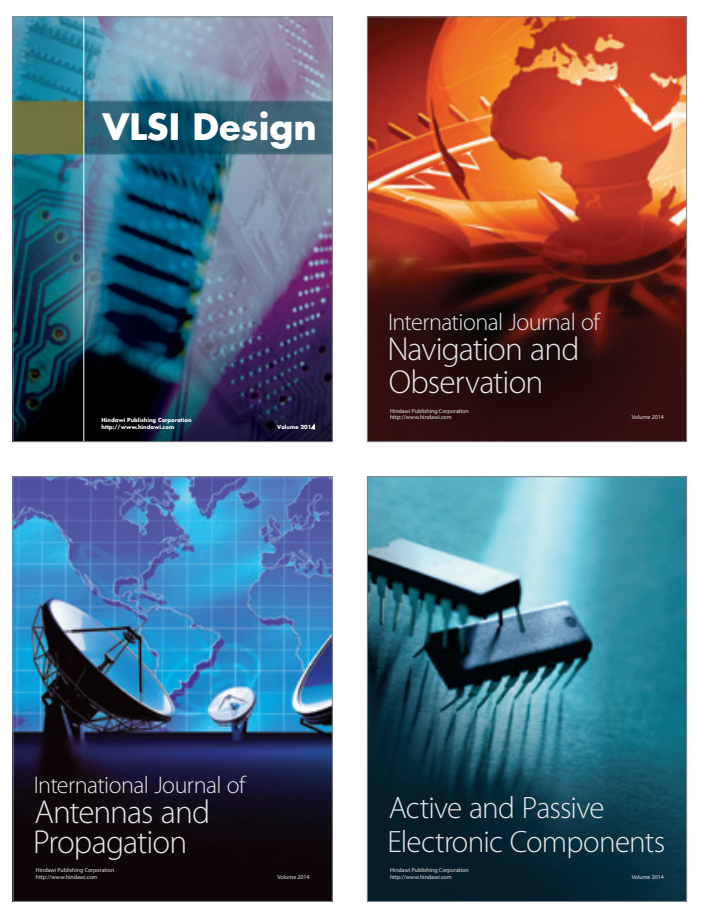
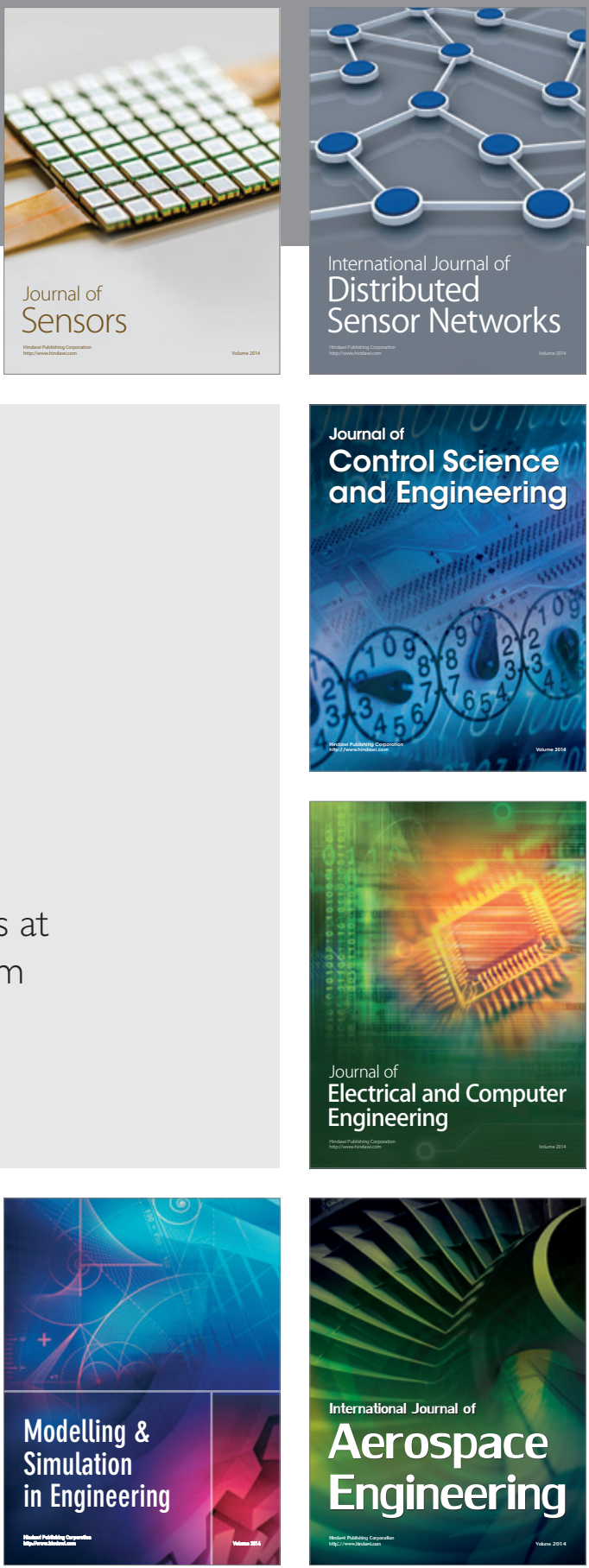

Journal of

Control Science

and Engineering
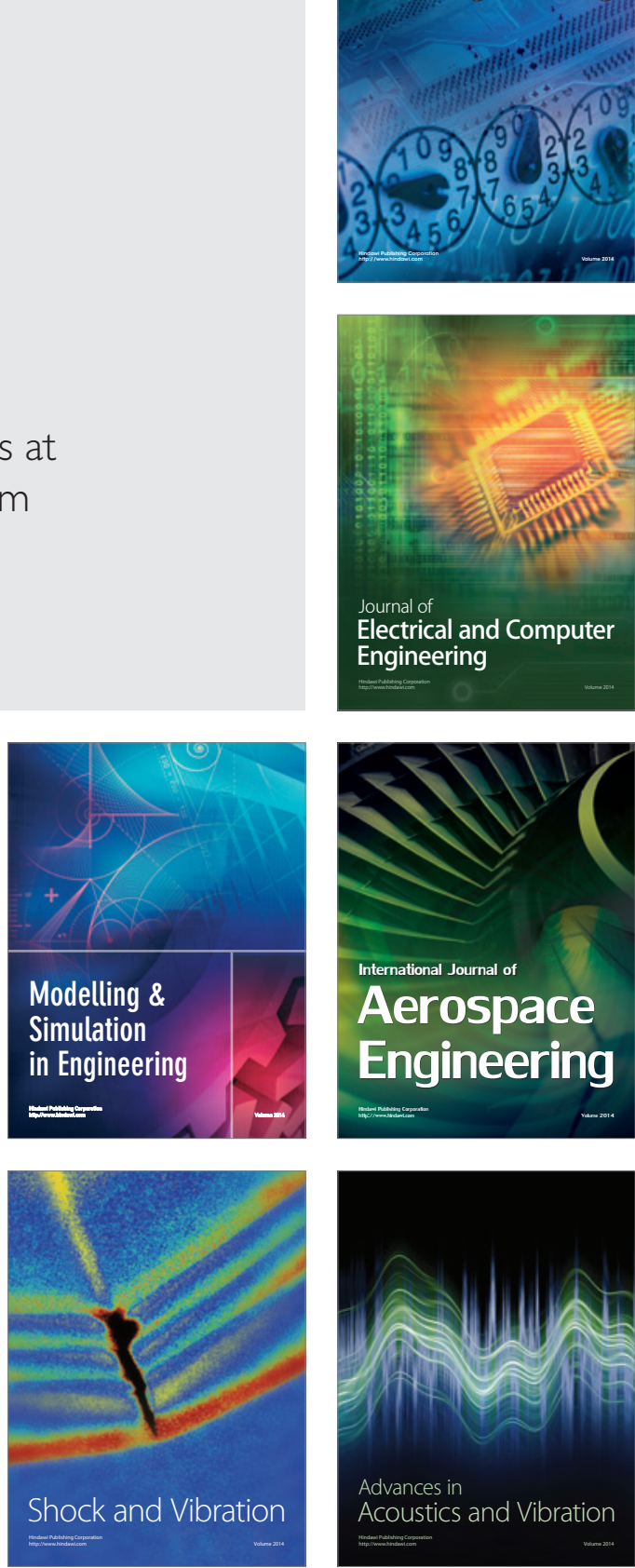